\title{
Dynamical Separation of Bulk and Edge Transport in HgTe-Based 2D Topological Insulators
}

\author{
Matthieu C. Dartiailh $\odot,{ }^{1}$ Simon Hartinger, ${ }^{2,3}$ Alexandre Gourmelon, ${ }^{1}$ Kalle Bendias, ${ }^{2,3}$ Hugo Bartolomei®, ${ }^{1}$ \\ Hiroshi Kamata $\odot,{ }^{1}$ Jean-Marc Berroir, ${ }^{1}$ Gwendal Fève, ${ }^{1}$ Bernard Plaçais, ${ }^{1}$ Lukas Lunczer, ${ }^{2,3}$ Raimund Schlereth $\odot,{ }^{2,3}$ \\ Hartmut Buhmann, ${ }^{2,3}$ Laurens W. Molenkamp, ${ }^{2,3}$ and Erwann Bocquillon $\oplus^{1}$ \\ ${ }^{1}$ Laboratoire de Physique de l'École Normale Supérieure, ENS, PSL Research University, CNRS, Sorbonne Université, \\ Université de Paris, 24 rue Lhomond, 75231 Paris Cedex 05, France \\ ${ }^{2}$ Physikalisches Institut (EP3), Am Hubland, Universität Würzburg, D-97074 Würzburg, Germany \\ ${ }^{3}$ Institute for Topological Insulators, Am Hubland, Universität Würzburg, D-97074 Würzburg, Germany
}

(Received 8 July 2019; accepted 13 January 2020; published 19 February 2020)

\begin{abstract}
Topological effects in edge states are clearly visible on short lengths only, thus largely impeding their studies. On larger distances, one may be able to dynamically enhance topological signatures by exploiting the high mobility of edge states with respect to bulk carriers. Our work on microwave spectroscopy highlights the response of the edges which host very mobile carriers, while bulk carriers are drastically slowed down in the gap. Though the edges are denser than expected, we establish that charge relaxation occurs on short timescales and suggest that edge states can be addressed selectively on timescales over which bulk carriers are frozen.
\end{abstract}

DOI: 10.1103/PhysRevLett.124.076802

Promising platforms have emerged to investigate the physics of two-dimensional (2D) topological insulators, which exhibit the quantum spin Hall (QSH) effect. Prominently, HgTe quantum wells (QWs) have revealed many transport signatures of the QSH effect [1-4], or a fractional Josephson effect, a signature of topological superconductivity [5,6], in $\mathrm{HgTe}$-based Josephson junctions. Another promising material system, InAs/GaSb double QWs, also shows the quantized conductance that accompanies topological edge state transport $[7,8]$ while several layered materials are currently under development, such as bismuthene [9] or $\mathrm{WTe}_{2}$ [10]. However, signatures of topology are in transport at most visible on a few microns despite the expected topological protection $[1,8,11]$. A significant reason is the presence of bulk bands, which naturally form puddles near the gap. Though these states poorly conduct, they may introduce scattering of the topological states $[12,13]$. The associated density of states (DOS) is large, especially in HgTe QWs with indirect band gap.

Topological signatures may be enhanced in dynamical studies by exploiting the difference in transport or scattering timescales between topological and bulk carriers. Recent efforts have demonstrated that dynamical

Published by the American Physical Society under the terms of the Creative Commons Attribution 4.0 International license. Further distribution of this work must maintain attribution to the author(s) and the published article's title, journal citation, and DOI. experiments in the $\mathrm{THz}$ range [14] or using time-resolved ARPES [15] allow the investigation and manipulation of topological states despite bulk background carriers. Transposing this idea to transport, we propose microwave drives to dynamically isolate quasiballistic topological edge modes while bulk carriers have an evanescent contribution since their diffusivity collapses in the gap. Besides, unlike ARPES or $\mathrm{THz}$ studies, microwave measurements can be operated in situ, in devices with top gates and/or protective capping layers, relevant for emerging topological electronics.

Here, we report on a systematic study of microwave spectroscopy, performed at a temperature of $T=10 \mathrm{~K}$ in a cryogenic probe station, in the frequency range $10 \mathrm{MHz}-$ $10 \mathrm{GHz}$, to investigate narrow $\mathrm{HgTe}$ QWs. Microwave signals enable simultaneous measurements of the capacitive response, sensitive to the density of electronic states, and of the resistive response, probing their ability to conduct, as exemplified recently in three-dimensional topological insulators [16-19]. Microwave capacitance spectroscopy thus naturally evidences the resulting $R C$ charge relaxation times of each transport channel. We combine this information with geometric scalings and gate control of the electron density. In this Letter, we show that the conduction and valence bands of the $\mathrm{QW}$ are ruled by a single-mode dynamics, corresponding to bulk carriers in good agreement with $\mathbf{k} \cdot \mathbf{p}$ band structure predictions. In contrast, the microwave spectra confirm a striking twomode dynamics when the Fermi level approaches the gap. From their dynamical transport properties, the two modes can be ascribed to bulk and edge carriers. This ascription is 
confirmed by geometric scalings [20]. We find that the edges host very mobile carriers with fast dynamics, though they are denser than theoretical prediction for helical edge states. In contrast, bulk states have high resistance and contribute an enormous DOS near the gap, resulting in a slow dynamics. Consequently, the charge relaxation frequencies associated to bulk and edge carriers differ by more than 1 order of magnitude, thus paving the way for more robust experiments in which topological edges can be dynamically and selectively addressed, while bulk carriers remain frozen.

Samples.-Our samples are based on $\mathrm{HgTe} / \mathrm{HgCdTe}$ QWs grown by molecular beam epitaxy on CdTe substrates [see Fig. 1(a)]. The thickness $t$ of the QWs varies between 5 and $11 \mathrm{~nm}$. The protective $\mathrm{HgCdTe}$ capping layer has a typical thickness of $15 \mathrm{~nm}$. In these QWs, the band structure consists of light electrons in the conduction band and heavier holes in the valence band. A topological phase transition occurs for the critical thickness $t_{c} \simeq 6.4 \mathrm{~nm}$. For $t>t_{c}$, an inverted band ordering enforces the appearance of topologically protected QSH edge states in the gap of the QWs [21]. For $t<t_{c}$, the band ordering is normal and no edge states occur; the sample is topologically trivial. The QWs are first characterized using standard Hall-bar measurements, yielding a mobility around $2 \times 10^{5} \mathrm{~cm}^{2} \mathrm{~V}^{-1} \mathrm{~s}^{-1}$ (measured at a density $3 \times 10^{11} \mathrm{~cm}^{-2}$ in the conduction band). Multiple QWs have been investigated and have given similar results. This Letter focuses on several devices realized on one layer, with a thickness of $8 \mathrm{~nm}$ and a predicted gap around $8 \mathrm{meV}$ (a)

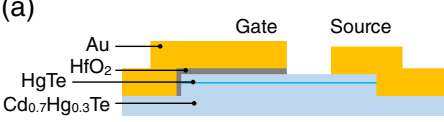

(c)

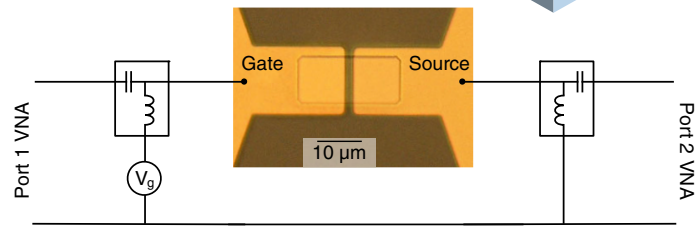

(d)

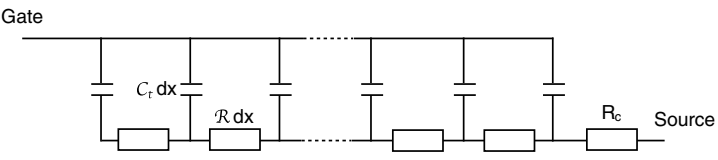

FIG. 1. Experimental techniques: (a) schematic side view of the device, showing the layers of the $\mathrm{HgTe} / \mathrm{HgCdTe}$ heterostructure, the gate, and the contact. (b) Artist view of the device, embedded in a coplanar waveguide. (c) High-frequency measurement setup, based on a vector network analyzer (VNA), the ports of which are connected to source and gate. Two bias tees enable to apply the dc gate voltage $V_{g}$ controlling the electron density. (d) Model of the capacitor as a distributed $R C$ line, with a contact resistance $R_{c}$, a line resistance $\mathcal{R}$, and a line capacitance $\mathcal{C}_{t}$. [see Fig. 2(a)]. Additional data obtained on other topological and trivial (noninverted) samples are reported in the Supplemental Material [20]. The devices comprise square capacitors of side $L=3,5,10,20 \mu \mathrm{m}$, embedded in gold coplanar waveguides [Figs. 1(a) and 1(b)]. The capacitor mesa is defined via a wet-etching technique [11] that preserves the high crystalline quality and the high mobility of the epilayer. The contacts and gate are patterned via optical lithography. The gold gate electrode is evaporated on top of a 7-nm-thick $\mathrm{HfO}_{2}$ insulating layer, grown by lowtemperature atomic layer deposition [11]. An ohmic contact is deposited by Au evaporation.

Admittance measurements. - The capacitors are measured in a cryogenic rf probe station at a temperature $T=$ $10 \mathrm{~K}$ using a vector network analyzer over three decades of frequency $10 \mathrm{MHz}<f<10 \mathrm{GHz}$. Standard in situ calibration techniques enable to de-embed the response of the circuitry from the admittance $Y(\omega)$ of interest, with $\omega=2 \pi f$. Earlier works $[18,19,22]$ have demonstrated that the capacitors can be described (assuming translation invariance along the transverse direction) by a distributed $R C$ line of length $L$, with line capacitance $\mathcal{C}_{t}$ and resistance $\mathcal{R}$, such as the one depicted in Fig. 1(d). At the lowest order in frequency, the device is equivalent to a capacitor $C_{t}=\mathcal{C}_{t} L$, with the admittance given by $Y(\omega)=i \omega C_{t}$. The total capacitance $C_{t}$ is the series addition of the geometric capacitance $C_{g}$ and the quantum capacitance $C_{q}$, which accounts for the increase of the Fermi energy and reads $C_{q}=e^{2} \rho$, where $\rho$ is the DOS (for weakly interacting systems in the limit of zero temperature). The geometric capacitance $C_{g}$ is extracted at high densities in the valence band for which $C_{t} \simeq C_{g}$ (see $[18,20,22]$ ). Since the $\mathrm{QW}$ is never depleted even in the gap, the geometry remains that of a plate capacitor. We thus calculate $C_{q}$ assuming a

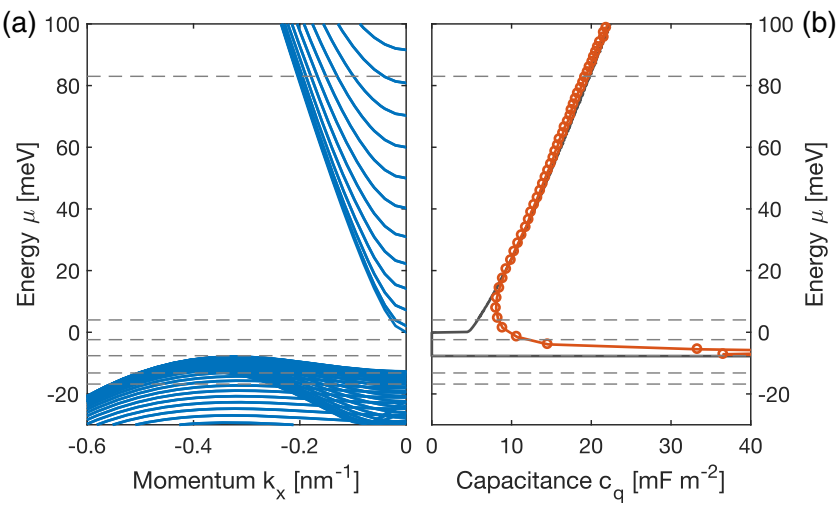

FIG. 2. Quantum capacitance of HgTe quantum wells: (a) energy bands as function of momentum $k_{x}$, as calculated for different values of transverse momentum $k_{y}$. (b) Quantum capacitance $c_{q}$ (per unit area) as function of energy $\mu$ (for a size of $L=10 \mu \mathrm{m})$. The gray line corresponds to the computed value of $c_{q}$ given the $\mathbf{k} \cdot \mathbf{p}$ band structure shown in panel (a), the red dots and line to experimental data. 
constant $C_{g}$ and compute the chemical potential [23] $\mu=$ $\int V_{g}\left\{1-\left[C_{t}(V) / C_{g}\right]\right\} d V$ to allow for comparison with predictions obtained from $\mathbf{k} \cdot \mathbf{p}$ band structure calculations $24,25]]$. The origin of the chemical potential is fixed to the maximum of the sample sheet resistance [20].

Quantum capacitance.-Figure 2 shows experimental data of the (areal) quantum capacitance $c_{q}(\mu)=C_{q}(\mu) / L^{2}$ and $\mathbf{k} \cdot \mathbf{p}$ predictions. The capacitance is low in the conduction band $\left(c_{q}<20 \mathrm{mF} \mathrm{m}^{-2}\right.$ for $\left.\mu>0\right)$ and increases quasi-linearly with $\mu$, as expected for two-dimensional bands with quasilinear band dispersion. The agreement with $\mathbf{k} \cdot \mathbf{p}$ calculations is excellent in this regime, provided the value of $C_{g}$ is fine-tuned (within $\pm 2 \%$ ). In this regime, the quantum capacitance is given by [18] $c_{q}=$ $e^{2} \mu /\left[\pi\left(\hbar v_{F}\right)^{2}\right]$, with the Fermi velocity $v_{F} \simeq 1 \times 10^{6} \mathrm{~ms}^{-1}$. In the valence band $(\mu \lesssim 0)$, we observe that $c_{q}$ is very high $\left(c_{q}>40 \mathrm{mF} \mathrm{m}^{-2}\right)$ as the valence band has a high mass. $c_{q}$ is however difficult to extract with high precision in this range, due to the finite value of the geometric capacitance (here $c_{g}=3.91 \mathrm{mF} \mathrm{m}^{-2} \pm 2 \%$ ). In between these two regimes, a minimum is observed in the data $c_{q} \simeq 10 \mathrm{mF} \mathrm{m}^{-2}$. It indicates the gap of the QW, where QSH edge states should be observed. A modeling as merely one-dimensional edge states yields a quantum capacitance per unit length of $\left(4 e^{2} / h v_{F}\right) \simeq 0.2 \mathrm{nF} \mathrm{m}^{-1}$ for the topological edge states
[26,27]. On a $L=10 \mu \mathrm{m}$ device, it corresponds to a very low areal contribution $c_{q}<0.1 \mathrm{mF} \mathrm{m}^{-2}$, much smaller than the observed minimum. This residual contribution in the gap is thus unlikely to originate solely from the edge states, but more likely from disorder in the bulk bands. The smearing is much larger than the temperature $(k T \lesssim 1 \mathrm{meV})$ or any broadening induced by the probe signal, and may be attributed to inhomogeneous bulk bands, charge puddles due to disorder, thickness fluctuations [28], or electrostatics. As a consequence of the extremely large DOS of the valence band, the minimum of $c_{q}$ is surprisingly upshifted in energy with respect to the theoretically predicted gap (here typically around $\mu=10 \mathrm{meV}$ ).

Edge and bulk dynamics.-For higher frequencies, dissipative and propagative effects set in. As depicted in Fig. 1(d), dissipation results from the access resistance $R_{c}$ and the finite sheet resistance $R=\mathcal{R} L$ of the HgTe. For frequencies exceeding the charge relaxation frequency $1 / 2 \pi R C_{t}$ of the device, typically $1 \mathrm{GHz}$ for $L=10 \mu \mathrm{m}$, the capacitors host evanescent waves [18,22,29], driven by the resistance of the HgTe film. Assuming translation invariance along the transverse axis, the admittance of the capacitor reads [20]

$$
Y(\omega)=\frac{1}{R_{c}+\frac{R}{i k \tanh (i k)},}
$$
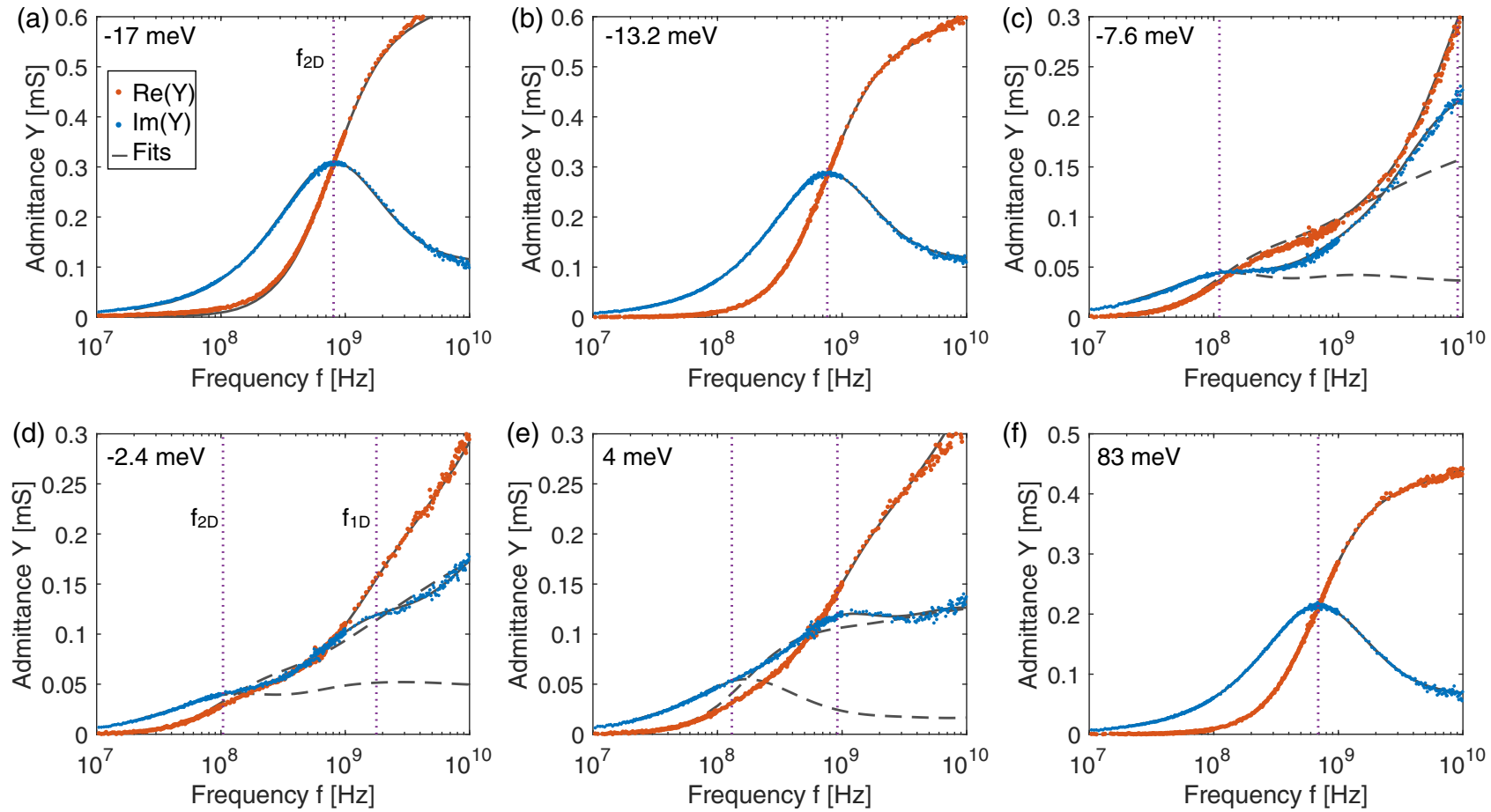

FIG. 3. High-frequency admittance: admittance $Y$ as function of frequency $f$ for different energies $\mu$ (indicated in each panel; see also Fig. 2) for $L=5 \mu \mathrm{m}$. Panels (a),(b) correspond to the valence band, (f) to the conduction band, and show a good agreement with a single-mode model (plain gray line). The other panels (c)-(e) correspond to the transition from valence to conduction bands. They are only well fitted by the two-mode model (plain gray line), while single-mode models fail (dashed gray line). The vertical dotted lines indicate the values of the charge relaxation frequencies $f_{2 \mathrm{D}}$ and $f_{1 \mathrm{D}}$. 
where $R_{c}$ describes the lumped contact resistance, while the tanh term describes the evanescent waves in the capacitor, with $k=\sqrt{i R C_{t} \omega}$.

The fits obtained with this simple model are in excellent agreement in a wide range of energies corresponding to the valence and conduction bands [as illustrated in Figs. 3(a) and 3(f)] (see also the Supplemental Material [20] for linear scale and Nyquist plots) and confirm the validity of the model. In particular, the crossover between the lowfrequency and evanescent regime is highlighted by a clear maximum in $\operatorname{Im}[Y(\omega)]$. The fit yields the value of the $\mathrm{HgTe}$ sheet resistance $R$ and of the contact resistance $R_{c}$. $R_{c}$ is found to be relatively independent of $V_{g}$ in the validity range of the model, with $R_{c}=0.3-4 \mathrm{k} \Omega$ depending on the device. However, we observe strong discrepancies arising near the gap between this simple one-carrier model and the data as illustrated in Figs. 3(c)-3(e). In particular, the admittance $Y$ exhibits two successive low-frequency regimes (dc-0.1 GHz and $0.1-2 \mathrm{GHz}$ ). We attribute these features to the presence of two independent types of charge carriers, a priori ascribed to the bulk (2D) and edge (1D carriers), with different charge relaxation times. Consequently, we model the system using two parallel distinct distributed $R C$ circuits such that $Y(\omega)=Y^{1 \mathrm{D}}(\omega)+$ $Y^{2 \mathrm{D}}(\omega)$ where $Y^{1 \mathrm{D} / 2 \mathrm{D}}$ are both given by Eq. (1). We obtain an excellent agreement between model and data over three decades of frequency [Figs. 3(c)-3(e)] and the two lowfrequency regimes are fully captured. The results are shown in Fig. 4(a). The largest contribution $c_{q}^{2 \mathrm{D}}$ is attributed to the two-dimensional bulk states and is observed to follow the $\mathbf{k} \cdot \mathbf{p}$ predictions. The second contribution appears close to the gap and is consequently attributed to edge carriers $c_{q}^{1 \mathrm{D}}$. The above attribution of $c_{q}^{1 \mathrm{D} / 2 \mathrm{D}}$ is validated by a separate study of the length dependence of $c_{q}$ [20], which confirms the values of $c_{q}^{1 \mathrm{D} / 2 \mathrm{D}}$, their variations with $\mu$, and more importantly their one- or two-dimensional characters. The one-dimensional component can only be detected in inverted (topological) heterostructures; it is thus likely a signature of the topological edge states. However, $c_{q}^{1 \mathrm{D}} \simeq$ $10 \mathrm{nF} \mathrm{m}^{-1}$ remains 20-50 larger than that of a bare helical edge state, suggesting two plausible scenarios. First, in line with the large quantum capacitances which have been measured for quantum Hall edge states [30,31], our results could be attributed to edge reconstruction and formation of compressible stripes under the action of electrostatics and electron-electron interactions [32-34]. In a second more likely scenario, our observations could originate from the presence of residual charge puddles near the edges, to which the edge states could couple. These puddles result from disorder, especially when the gap of the QW is small, and may be enhanced on the edges by electrostatics as the topological edge states themselves may screen the action of the gate. Regardless of the microscopic details, a (spindegenerate) subband, could contribute up to $5 \mathrm{nFm}^{-1}$, so that typically 1-2 puddle states would reside in the vicinity of the topological edge states, introducing some scattering [12] in the edge states.

Our measurement technique is primarily sensitive to capacitances. Consequently, though the contact and sheet resistances can easily be separated in the single-carrier model, they can only be determined with an accuracy of $50 \%$ in the two-carrier model. We observe nonetheless that, near the gap, the total 2D resistance $R_{t}^{2 \mathrm{D}} \gtrsim 40 \mathrm{k} \Omega$ is much larger than the total $1 \mathrm{D}$ resistance $R_{t}^{1 \mathrm{D}} \simeq 10-15 \mathrm{k} \Omega$. In an ideal QSH regime, the geometry resembles that of a mesoscopic capacitor [35-37], and $R_{t}^{1 \mathrm{D}}$ should be dominated by a contact resistance, between $R_{K} / 4$ (coherent regime) and $R_{K} / 2$ (incoherent regime) [38,39], where $R_{K}=\left(h / e^{2}\right)$ is the resistance quantum. Though no quantized resistance plateau is observed in our samples, the extracted values of $R_{t}^{1 \mathrm{D}}$ tend to validate the topological origin of the $1 \mathrm{D}$ contribution. Higher resistance $\left(\lesssim R_{K}\right)$ are observed in some devices, probably due to the presence of scattering as the perimeters of the sample are large ( $\simeq 15 \mu \mathrm{m}$ here). This observation justifies a posteriori the description of the edge carriers as a mixed contact and line resistances.

Charge relaxation frequencies. - This broadband analysis also yields the charge relaxation frequencies given by $f_{1 \mathrm{D} / 2 \mathrm{D}}=1 / 2 \pi R_{t}^{1 \mathrm{D} / 2 \mathrm{D}} C_{t}^{1 \mathrm{D} / 2 \mathrm{D}}$ as plotted in Fig. 4(b). In the conduction and valence bands, where the single-mode picture holds, we observe that the (bulk) electrons have a high response frequency $\left(f_{2 \mathrm{D}} \simeq 0.7-0.8 \mathrm{GHz}\right)$. However, this charge relaxation frequency decreases drastically when the Fermi level approaches the gap and reaches $f_{2 \mathrm{D}} \simeq 0.1 \mathrm{GHz}$. In the same energy range, the edge electrons appear very mobile, with $f_{1 \mathrm{D}} \simeq 0.9 \mathrm{GHz}$. The dynamics thus clearly highlights the presence of mobile edge carriers near the gap in an energy range where bulk carriers are much slower. At high frequency, bulk transport is evanescent over a length $\delta_{2 \mathrm{D}} \simeq L \sqrt{\left(2 f_{2 \mathrm{D}} / f\right)} \ll L$, whereas edge currents penetrate over a larger distance $\left(\delta_{2 \mathrm{D}} \ll \delta_{1 \mathrm{D}} \sim L\right)$. The analysis also confirms that topological carriers coexist with the conduction band over a significant range of energies $(\simeq 40 \mathrm{meV}$, indicated by the shaded area in Fig. 4), in line with theoretical predictions [40] and observations [5,41].

The edge and bulk signals are thus clearly distinguished in the microwave spectra, via the charge relaxation frequency, while they are intertwined in measurements of the resistance or quantum capacitance at low frequency. Beyond this mere identification, the large difference between $f_{1 \mathrm{D}}$ and $f_{2 \mathrm{D}}$ (about a decade) also has strong implications: it demonstrates that a careful (and geometrydependent) choice of the drive frequency $f_{2 \mathrm{D}} \ll f$ would allow for addressing dynamically the edge states on timescales over which the bulk states are frozen. Near the bottom of the conduction $(\mu \simeq 0 \mathrm{meV})$, exciting on one end of the device of length $L$ at frequency 

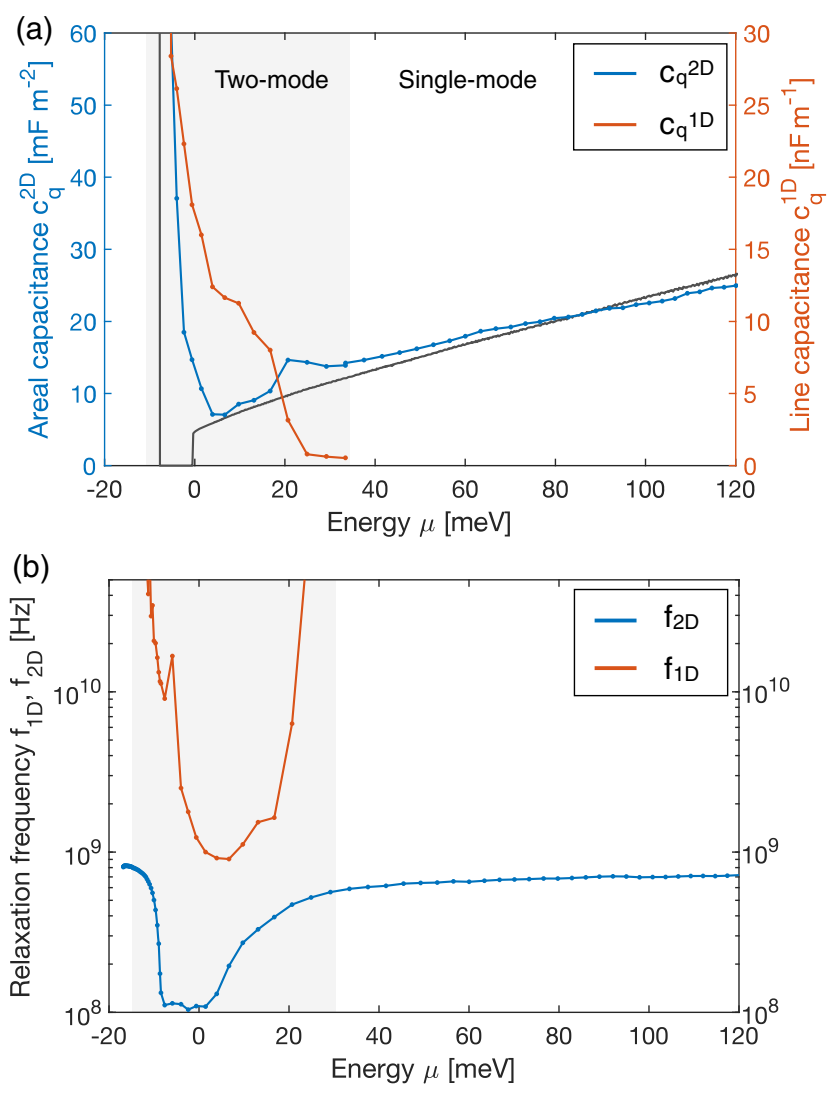

FIG. 4. Quantum capacitances and charge relaxation frequencies: (a) on the left axis, for $L=5 \mu \mathrm{m}$, the quantum capacitance $c_{q}^{2 \mathrm{D}}$ attributed to the $2 \mathrm{D}$ bulk carriers is shown as a blue line as a function of energy $\mu$, together with the prediction of the $\mathbf{k} \cdot \mathbf{p}$ calculations (solid gray line). The $1 \mathrm{D}$ contribution $c_{q}^{1 \mathrm{D}}$ is plotted as a red line on the right axis. (b) The relaxation frequencies $f_{2 \mathrm{D}}$ and $f_{1 \mathrm{D}}$ are plotted as function of energy $\mu$, as blue and red lines, respectively.

$f=2 f_{1 \mathrm{D}} \simeq 20 f_{2 \mathrm{D}}$ would for example result in an increase of the ratio of topological and bulk signals at the other end of the device by a significant factor $e^{-L / \delta_{1 \mathrm{D}}} / e^{-L / \delta_{2 \mathrm{D}}}=e^{\sqrt{\left(f / 2 f_{2 \mathrm{D}}\right)}-\sqrt{\left(f / 2 f_{1 \mathrm{D}}\right)}} \simeq 9$.

Summary and outlook.-As a conclusion, our measurements bring up new information on the transport of topological edge states. While the bulk is mostly insulating in the gap, its DOS remains rather large. Nonetheless, the edge response can be isolated in high-frequency admittance. They are rather dense, suggesting that the topological edge states are surrounded by additional states contributing to the DOS and to scattering, but not to transport. Their nature remains to be clarified in further studies. Importantly, charge relaxation frequencies for bulk and edge states differ typically by a decade. Consequently, microwaves (typically in the $\mathrm{GHz}$ range) could be utilized to selectively address topological edge transport irrespective of the presence of bulk states. Enhanced topological signatures on larger scales could for example reveal Luttinger liquid physics [39,42-45] or dynamical spin and charge transport [46-48].
Finally, we argue that the method is applicable to other topological phases (3D topological insulators or Weyl semimetals) where topological transport often competes with numerous less mobile trivial modes.

The data sets generated and/or analyzed during the current study are available from the corresponding author on reasonable request.

This work has been supported by the ERC, under Contracts No. ERC-2017-StG "CASTLES" and No. ERC-2017-Adv "4TOPS", the Domaine d'Intérêt Majeur SIRTEQ, under contract " $\mu$ QS", the DFG (SPP 1666, SFB 1170, and Leibniz Program), and the Bavarian Ministry of Education (ENB and ITI). We gratefully acknowledge insightful discussions with D. Bercioux, C. Brüne, D. Carpentier, M. R. Calvo, and T. van der Berg. M. C. D., S. H., A. G., and H. B. performed the measurements. S. H. and K. B. fabricated the samples, based on MBE layers grown by R.S., L. L. and E. B. supervised the project. All authors participated to the analysis of the results and to the writing of the Letter.

[1] M. Konig, S. Wiedmann, C. Brune, A. Roth, H. Buhmann, L. W. Molenkamp, X.-L. Qi, and S.-C. Zhang, Science 318, 766 (2007).

[2] A. Roth, C. Brune, H. Buhmann, L. W. Molenkamp, J. Maciejko, X.-L. Qi, and S.-C. Zhang, Science 325, 294 (2009).

[3] C. Brüne, A. Roth, H. Buhmann, E. M. Hankiewicz, L. W. Molenkamp, J. Maciejko, X.-L. Qi, and S.-C. Zhang, Nat. Phys. 8, 485 (2012).

[4] M. R. Calvo, F. de Juan, R. Ilan, E. J. Fox, A. J. Bestwick, M. Mühlbauer, J. Wang, C. Ames, P. Leubner, C. Brüne, S. C. Zhang, H. Buhmann, L. W. Molenkamp, and D. Goldhaber-Gordon, Phys. Rev. Lett. 119, 226401 (2017).

[5] E. Bocquillon, R. S. Deacon, J. Wiedenmann, P. Leubner, T. M. Klapwijk, C. Brüne, K. Ishibashi, H. Buhmann, and L. W. Molenkamp, Nat. Nanotechnol. 12, 137 (2017).

[6] R. S. Deacon, J. Wiedenmann, E. Bocquillon, F. Domínguez, T. M. Klapwijk, P. Leubner, C. Brüne, E. M. Hankiewicz, S. Tarucha, K. Ishibashi, H. Buhmann, and L. W. Molenkamp, Phys. Rev. X 7, 021011 (2017).

[7] I. Knez, R.-R. Du, and G. Sullivan, Phys. Rev. Lett. 107, 136603 (2011).

[8] L. Du, I. Knez, G. Sullivan, and R.-R. Du, Phys. Rev. Lett. 114, 096802 (2015).

[9] F. Reis, G. Li, L. Dudy, M. Bauernfeind, S. Glass, W. Hanke, R. Thomale, J. Schäfer, and R. Claessen, Science 357, 287 (2017).

[10] S. Wu, V. Fatemi, Q. D. Gibson, K. Watanabe, T. Taniguchi, R. J. Cava, and P. Jarillo-Herrero, Science 359, 76 (2018).

[11] K. Bendias, S. Shamim, O. Herrmann, A. Budewitz, P. Shekhar, P. Leubner, J. Kleinlein, E. Bocquillon, H. Buhmann, and L. W. Molenkamp, Nano Lett. 18, 4831 (2018).

[12] J. I. Väyrynen, M. Goldstein, Y. Gefen, and L. I. Glazman, Phys. Rev. B 90, 115309 (2014). 
[13] L. Lunczer, P. Leubner, M. Endres, V. L. Müller, C. Brüne, H. Buhmann, and L. W. Molenkamp, Phys. Rev. Lett. 123, 047701 (2019).

[14] L. Luo, X. Yang, X. Liu, Z. Liu, C. Vaswani, D. Cheng, M. Mootz, X. Zhao, Y. Yao, C.-Z. Wang, K.-M. Ho, I. E. Perakis, M. Dobrowolska, J. K. Furdyna, and J. Wang, Nat. Commun. 10, 607 (2019).

[15] H. Soifer, A. Gauthier, A. F. Kemper, C. R. Rotundu, S.-L. Yang, H. Xiong, D. Lu, M. Hashimoto, P. S. Kirchmann, J. A. Sobota, and Z.-X. Shen, Phys. Rev. Lett. 122, 167401 (2019).

[16] S. Xu, Y. Han, X. Chen, Z. Wu, L. Wang, T. Han, W. Ye, H. Lu, G. Long, Y. Wu, J. Lin, Y. Cai, K. M. Ho, Y. He, and N. Wang, Nano Lett. 15, 2645 (2015).

[17] D. A. Kozlov, D. Bauer, J. Ziegler, R. Fischer, M. L. Savchenko, Z. D. Kvon, N. N. Mikhailov, S. A. Dvoretsky, and D. Weiss, Phys. Rev. Lett. 116, 166802 (2016).

[18] A. Inhofer, S. Tchoumakov, B. A. Assaf, G. Fève, J. M. Berroir, V. Jouffrey, D. Carpentier, M. O. Goerbig, B. Plaçais, K. Bendias, D. M. Mahler, E. Bocquillon, R. Schlereth, C. Brüne, H. Buhmann, and L. W. Molenkamp, Phys. Rev. B 96, 195104 (2017).

[19] A. Inhofer, J. Duffy, M. Boukhicha, E. Bocquillon, J. Palomo, K. Watanabe, T. Taniguchi, I. Estève, J. M. Berroir, G. Fève, B. Plaçais, and B. A. Assaf, Phys. Rev. Appl. 9, 024022 (2018).

[20] See Supplemental Material at http://link.aps.org/ supplemental/10.1103/PhysRevLett.124.076802 for additional data and analysis of topological and trivial devices.

[21] B. A. Bernevig, T. L. Hughes, and S.-C. Zhang, Science 314, 1757 (2006).

[22] E. Pallecchi, A. C. Betz, J. Chaste, G. Fève, B. Huard, T. Kontos, J.-M. Berroir, and B. Plaçais, Phys. Rev. B 83, 125408 (2011).

[23] C. N. Berglund, IEEE Trans. Electron Devices ED-13, 701 (1966).

[24] C. R. Becker, V. Latussek, A. Pfeuffer-Jeschke, G. Landwehr, and L. W. Molenkamp, Phys. Rev. B 62, 10353 (2000).

[25] E. G. Novik, A. Pfeuffer-Jeschke, T. Jungwirth, V. Latussek, C. R. Becker, G. Landwehr, H. Buhmann, and L. W. Molenkamp, Phys. Rev. B 72, 035321 (2005).

[26] J. Chaste, L. Lechner, P. Morfin, G. Fève, T. Kontos, J.-M. Berroir, D. C. Glattli, H. Happy, P. Hakonen, and B. Plaçais, Nano Lett. 8, 525 (2008).

[27] M. V. Entin and L. Braginsky, Phys. Rev. B 96, 115403 (2017).
[28] G. Tkachov, C. Thienel, V. Pinneker, B. Buettner, C. Bruene, H. Buhmann, L. W. Molenkamp, and E. M. Hankiewicz, Phys. Rev. Lett. 106, 076802 (2011).

[29] H. Graef, D. Mele, M. Rosticher, L. Banszerus, C. Stampfer, T. Taniguchi, K. Watanabe, E. Bocquillon, G. Fève, J.-M. Berroir, E. H. T. Teo, and B. Plaçais, J. Phys. 1, 01LT02 (2018).

[30] S. Takaoka, K. Oto, H. Kurimoto, K. Murase, K. Gamo, and S. Nishi, Phys. Rev. Lett. 72, 3080 (1994).

[31] S. Takaoka, K. Oto, S. Uno, K. Murase, F. Nihey, and K. Nakamura, Phys. Rev. Lett. 81, 4700 (1998).

[32] D. B. Chklovskii, B. I. Shklovskii, and L. I. Glazman, Phys. Rev. B 46, 4026 (1992).

[33] C. de C. Chamon and X. G. Wen, Phys. Rev. B 49, 8227 (1994).

[34] J. Wang, Y. Meir, and Y. Gefen, Phys. Rev. Lett. 118, 046801 (2017).

[35] M. Büttiker, H. Thomas, and A. Prêtre, Phys. Lett. A 180, 364 (1993).

[36] J. Gabelli, G. Fève, J.-M. Berroir, B. Plaçais, A. Cavanna, B. Etienne, Y. Jin, and C. Glattli, Science 313, 499 (2006).

[37] J. Gabelli, G. Fève, J.-M. Berroir, and B. Plaçais, Rep. Prog. Phys. 75, 126504 (2012).

[38] S. E. Nigg and M. Buttiker, Phys. Rev. B 77, 085312 (2008).

[39] T. Müller, R. Thomale, B. Trauzettel, E. Bocquillon, and O. Kashuba, Phys. Rev. B 95, 245114 (2017).

[40] X. Dai, T. L. Hughes, X.-L. Qi, Z. Fang, and S.-C. Zhang, Phys. Rev. B 77, 125319 (2008).

[41] K. C. Nowack, E. M. Spanton, M. Baenninger, M. König, J. R. Kirtley, B. Kalisky, C. Ames, P. Leubner, C. Brüne, H. Buhmann, L. W. Molenkamp, D. Goldhaber-Gordon, and K. A. Moler, Nat. Mater. 12, 787 (2013).

[42] C. Wu, B. A. Bernevig, and S.-C. Zhang, Phys. Rev. Lett. 96, 106401 (2006).

[43] A. Calzona, M. Carrega, G. Dolcetto, and M. Sassetti, Phys. Rev. B 92, 195414 (2015).

[44] E. Bocquillon, V. Freulon, J.-M. Berroir, P. Degiovanni, B. Plaçais, A. Cavanna, Y. Jin, and G. Fève, Nat. Commun. 4, 1839 (2013).

[45] H. Kamata, N. Kumada, M. Hashisaka, K. Muraki, and T. Fujisawa, Nat. Nanotechnol. 9, 177 (2014).

[46] A. Inhofer and D. Bercioux, Phys. Rev. B 88, 235412 (2013).

[47] P. P. Hofer and M. Büttiker, Phys. Rev. B 88, 241308 (2013).

[48] P. P. Hofer, H. Aramberri, C. Schenke, and P. A. L. Delplace, Europhys. Lett. 107, 27003 (2014). 